Objectives: The objective of our work was to evaluate its prevalence and its different aspects.

Methods: A retrospective study including 11 files of GPA patients followed over a period of 10 years in The Internal Medecine Department at Tahar Sfar Hospital Mahdia TUNISIA.

Results: Neurological manifestations were reported in 5 patients: a pyramidal syndrome was noted in 3 patients with a normal cerebral MRI, a disabled headache was described in one patient with a normal cerebral MRI. Peripheral neuropathy in 2 cases, and the Electroneuromyogram objectified a mononeuropathy affecting the ulnar nerve, peroneal nerve, and musculocutaneous nerve in one patient and a distal sensitivomotor poly neuropathy in one patient. Cranial nerves damage was noted in two cases, one VII (1 case) and one V (1 case).

Conclusion: Our study results, show the importance of timely diagnosis of these neurological manifestations, as their evolution will depend on the early GPA treatment.

REFERENCES:

[1] doi: 10.1016/j.autrev.2017.07.007

Disclosure of Interests: None declared

DOI: 10.1136/annrheumdis-2021-eular.1660

\section{AB0769 EROSIVE SYNOVITIS, DACTYLITIS, HYPERCALCAEMIA, LYMPHADENOPATHY, ELEVATED SERUM ACE \& HYPOTHYROIDISM - AN UNUSUAL PRESENTATION OF IGG4-RELATED DISEASE}

E. Tranah ${ }^{1}$, A. Khan2, N. Bhadauria ${ }^{1}$, D. Mukerjee ${ }^{1} .{ }^{1}$ North Middlesex University Hospital, Rheumatology, London, United Kingdom; ${ }^{1}$ North Middlesex University Hospital, Rheumatology, London, United Kingdom

Background: IgG4-related disease (IgG4-RD) is a fibroinflammatory disorder which can affect almost any tissue in the body. We describe a challenging case of IgG4-RD which reflects the great heterogeneity of this disease.

Objectives: A 49-year-old Mauritanian gentleman was referred to Rheumatology with chronic progressive symmetrical synovitis affecting the upper and lower limbs. He had no features suggestive of a spondyloarthropathy or connective tissue disease. Blood tests revealed a raised C-reactive peptide (CRP) of $194 \mathrm{mg} / \mathrm{L}$ and erythrocyte sedimentation rate (ESR) of $46 \mathrm{~mm} /$ hr. His rheumatoid factor and anti-cyclic citrullinated peptide antibodies were negative. Anti-nuclear antibody was weakly positive at 1:80 with a speckled pattern, but extractable nuclear antigen antibodies, anti-double-strand DNA-antibodies and anti-neutrophil cytoplasm antibodies were negative. Complement proteins and creatine kinase were normal. Radiographs of the hands and feet demonstrated widespread erosive changes worst at the metacarpophalangeal, metatarsophalangeal and first tarsometatarsal joints, as well as swan-neck deformities of the fifth phalanges in both hands. Chest radiograph and serological virology screen were both unremarkable. He was subsequently diagnosed with a seronegative inflammatory arthritis and treated with intramuscular methylprednisolone. He was awaiting subcutaneous methotrexate.

Methods: He was hospitalised shortly after with worsening polyarthritis and hypercalcaemia $(3.34 \mathrm{mmol} / \mathrm{L})$. Examination demonstrated synovitis, new lymphadenopathy and dactylitis. Other investigations revealed a normocytic anaemia (Hb $80 \mathrm{~g} / \mathrm{dL}, \mathrm{MCV} 80 \mathrm{fL})$, neutrophilia $\left(20 \times 10^{9} / \mathrm{L}\right)$, eosinophilia $\left(4 \times 10^{9} / \mathrm{L}\right)$, thrombocythaemia $\left(500 \times 10^{9} / \mathrm{L}\right)$, hypoalbuminaemia $(25 \mathrm{~g} / \mathrm{L})$, CRP $200 \mathrm{mg} / \mathrm{L}$ and ESR $60 \mathrm{~mm} / \mathrm{hr}$. All immunoglobulins were raised with $\mathrm{IgG} 30.5 \mathrm{~g} / \mathrm{L}$. IgG4 subset was $3.0 \mathrm{~g} / \mathrm{L}$. Parathyroid hormone was low (< $0.7 \mathrm{pmol} / \mathrm{L})$ and vitamin $\mathrm{D}$ was $125 \mathrm{nmol} / \mathrm{L}$. Thyroid function, myeloma screen and blood tumour markers were unremarkable. Blood film revealed no blasts. Serum angiotensin-converting enzyme (ACE) was elevated at $171.9 \mathrm{U} / \mathrm{L}$. A CT-chest-abdomen-pelvis revealed an enlarged thyroid with retrosternal extension and widespread lymphadenopathy above the diaphragm.

Results: Biopsies of the thyroid gland and submandibular lymph node demonstrated lymphoplasmacytic fibroinflammatory changes and $>40 \%$ of IgG4 cells. Neither biopsy showed evidence of sarcoidosis, tuberculosis or malignancy. He was treated with a weaning regimen of high-dose oral corticosteroids resulting in normalisation of haematological, inflammatory and biochemical parameters. His musculoskeletal pain, swelling and early morning stiffness significantly improved too. Subcutaneous methotrexate was commenced and titrated upwards to $20 \mathrm{mg}$. Two months later, his arthritis relapsed upon reduction of corticosteroids. Again, there was a marked inflammatory response with raised CRP and ESR. He had also developed a new hypothyroidism (TSH > 100 $\mathrm{mU} / \mathrm{L}$, T4 $0.5 \mu \mathrm{g} / \mathrm{dL}$, positive TPO-antibody). Repeat hand and feet radiographs revealed severe erosive progressive disease (Figure 1). He awaits commencement of rituximab.

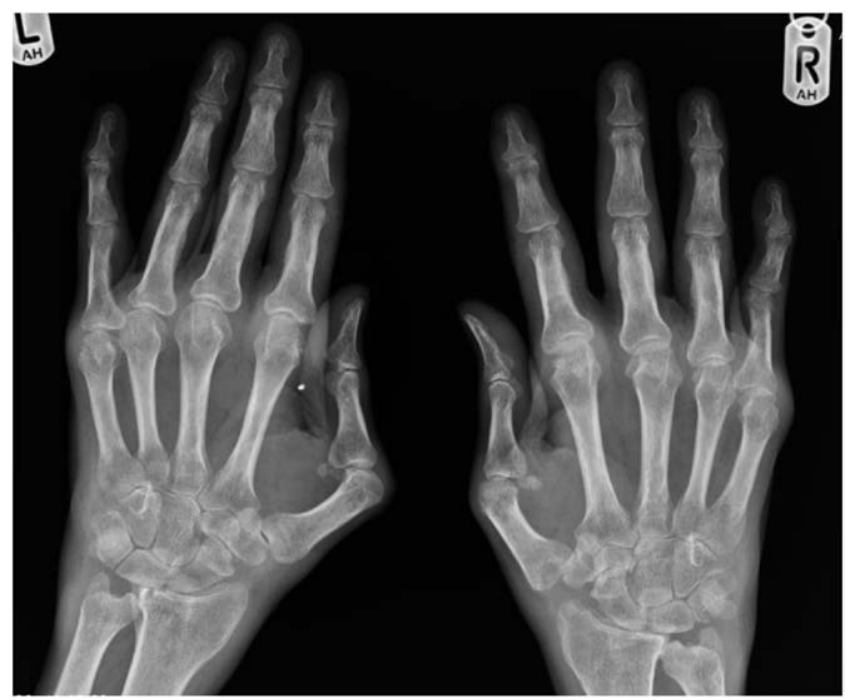

Figure 1. Plain hand radiograph demonstrating extensive erosive arthritis

Conclusion: This is a unique presentation for a rare disease. There are few case reports of IgG4-RD causing erosive synovitis with rheumatoid-like swan-neck deformities or hypercalcaemia. Furthermore, this is the first case report as far as we know, of the disease associated with dactylitis or a raised serum ACE. Prior to biopsy, our main working diagnoses were sarcoidosis or a lymphoproliferative malignancy. The peculiar presentation necessitated a second opinion via the national IgG4-RD MDT who confirmed the histological diagnosis of IgG4-RD. We hope that this interesting case highlights the importance of histology in diagnosis of complex cases. In addition, the case presentation adds to the multitude of clinical manifestations of IgG4-RD.

Disclosure of Interests: None declared

DOI: 10.1136/annrheumdis-2021-eular.1921

\section{AB0770 \\ A RARE CASE OF SYNOVIAL METASTASIS REVEALED BY KNEE PAIN}

M. Yasmine ${ }^{1}$, S. Miladi ${ }^{1}$, A. Fazaa ${ }^{1}$, S. Mariem ${ }^{1}$, K. Ouenniche ${ }^{1}$, L. Souebni ${ }^{1}$, S. Kassab ${ }^{1}$, S. Chekili ${ }^{1}$, K. Ben Abdelghani ${ }^{1}$, A. Laatar ${ }^{1} .{ }^{1}$ Mongi Slim Hospital, Rheumatology, Tunis, Tunisia

Background: Intra-articular masses are not frequently encountered in clinical practice. However, the differential diagnosis can be broad. Synovial metastasis is a rare presentation that carries a poor prognosis with a poor survival rate.

Objectives: Here by a case of synovial metastasis of the knee joint in a patient diagnosed with an adenocarcinoma of the lung.

Methods: A 60-year-old man with no remarkable past medical history presented to our department of rheumatology with right knee pain. He described a dull ache and swelling in his right knee with a dragging sensation, waking him up at night on a regular basis. The symptoms lasted from 6 months and were partially improved by analgesics and anti-inflammatory drugs. On examination, temperature was normal. The knee was edematous, erythematous, and warm with a range of motion of $<90^{\circ}$. Laboratory investigations showed high acute phase reactants, the erythrocyte sedimentation rate was $75 \mathrm{~mm}$. A plain radiograph of the left knee demonstrated a lytic lesion of the upper tibia. A magnetic resonance imaging of the right knee showed diffuse enlargement of the joint space due to a tissue infiltration within the synovium. These structures demonstrated heterogeneously increased T2 signal and intermediate T1 intensity characteristics. Most of the joint space was replaced by hyper enhancing synovium. At that time, differential considerations included severe inflammatory arthritis and synovial chondromatosis rather than unusual metastasis.

Results: Biopsy concluded to synovial metastasis from primary adenocarcinoma. Further investigations were necessary. Chest tomography showed a tumor process of left lower lobe associated with mediastinal lymphadenopathy. The patient was treated with palliative external radiotherapy to the right knee. The evolution was marked by the appearance of multiple tracheobronchial fistulas. The patient died 3 months later due to the progression of the disease. 
Conclusion: We report a rare case of synovial metastasis concomitant to a lung adenocarcinoma. It is important to make a rapid diagnosis as early recognition of malignant infiltration into joints ensure appropriate multidisciplinary management decisions. Disclosure of Interests: None declared DOI: 10.1136/annrheumdis-2021-eular.2143

\section{AB0771 $\quad$ HIGH DOSE INTRAVENOUS METHYLPREDNISOLONE INDUCES RAPID IMPROVEMENT OF VISUAL ACUITY IN NON-INFECTIOUS UVEITIS OF DIFFERENT IMMUNE MEDIATED INFLAMMATORY DISEASES}

N. Vegas-Revenga ${ }^{1}$, J. L. Martín-Varillas ${ }^{2}$, V. Calvo-Río ${ }^{3}$, I. González-Mazón ${ }^{3}$, L. Sanchez-Bilbao ${ }^{3}$, E. Beltrán ${ }^{4}$, A. Fonollosa ${ }^{5}$, O. Maiz-Alonso ${ }^{6}$, A. Blanco ${ }^{7}$, M. Cordero-Coma ${ }^{8}$, N. Ortego ${ }^{9}$, I. Torre-Salaberri ${ }^{10}$, F. Francisco ${ }^{11}$, S. Muñoz Fernandez $^{12}$, M. D. M. Esteban-Ortega ${ }^{13}$, M. Díaz-Llopis ${ }^{14}$, J. Cañal' ${ }^{15}, \mathrm{~J}$. A. Ventosa ${ }^{15}$, R. Demetrio-Pablo ${ }^{15}$, L. Domínguez ${ }^{16}$, M. Agudo-Bilbao ${ }^{3}$, S. Castañeda ${ }^{17}$, I. Ferraz-Amaro ${ }^{18}$, M. A. González-Gay ${ }^{3}$, R. Blanco ${ }^{3}$. ${ }^{1}$ Hospital Galdakao-Usansolo, Rheumatology, Galdakao, Spain; ${ }^{2}$ Hospital de Sierrallana, Rheumatology, Torrelavega, Spain; ${ }^{3}$ Hospital Universitario Marqués de Valdecilla, Rheumatology, Santander, Spain; ${ }^{4}$ Hospital Universitario Doctor Peset, Rheumatology, Valencia, Spain; ${ }^{5}$ Hospital Universitario de Cruces, Ophthalmology, Barakaldo, Spain; ${ }^{6}$ Hospital Universitario Donostia, Rheumatology, San Sebastian, Spain; ${ }^{7}$ Hospital Universitario Donostia, Ophthalmology, San Sebastian, Spain; ${ }^{8}$ Hospital de León, Ophthalmology, León, Spain; ${ }^{9}$ Hospital Universitario San Cecilio, Ophthalmology, Granada, Spain; ${ }^{10}$ Hospital Universitario de Basurto, Rheumatology, Bilbao, Spain; ${ }^{11} \mathrm{Hospital}$ Universitario de Gran Canaria Doctor Negrín, Rheumatology, Gran Canaria, Spain; ${ }^{12}$ Hospital Universitario Infanta Sofia, Rheumatology, Madrid, Spain; ${ }^{13} \mathrm{Hospital}$ Universitario Infanta Sofia, Ophthalmology, Madrid, Spain; ${ }^{14} \mathrm{Hospital}$ Universitario La Fe, Ophthalmology, Valencia, Spain; ${ }^{15}$ Hospital Universitario Marqués de Valdecilla, Ophthalmology, Santander, Spain; ${ }^{16} \mathrm{Hospital}$ Universitario San Agustín, Rheumatology, Avilés, Spain; ${ }^{17}$ Hospital Universitario de la Princesa, Rheumatology, Madrid, Spain; ${ }^{18} \mathrm{Hospital}$ Universitario de Canarias, Rheumatology, Canarias, Spain

Background: Rapid and effective remission-inducing therapy is mandatory in uveitis to avoid irreversible structural and functional damage. In some severe cases biological agents might be required (1-6).

High-dose intravenous methylprednisolone (IVMP) may achieve prompt control of inflammation in most immune mediated inflammatory diseases (IMID), including non-infectious uveitis (NIU).

Objectives: To evaluate the efficacy and safety of IVMP pulse therapy in NIU of different IMID.

Methods: Multicentre study of 71 patients with severe uveitis who received IVMP. The underlying diseases were: Vogt Koyanagy Harada disease (VKHD) $(n=24)$, Behçet disease (BD) (19), Sarcoidosis (5) and idiopathic NIU (23). The main outcome variable was Best-Corrected Visual Acuity (BCVA) estimated using the Snellen chart. BCVA that was assessed at 0 (basal), 2-5, 7, 15 and 30 days after IVMP.

The results are expressed as mean $\pm S D$ in normally distributed variables, or as median [IQR] when are not. Comparison of continuous variables was performed using the Wilcoxon test.

Results: We studied 46 $/ 25$ patients. The main features are shown in Table 1. IVMP dose ranged from 250 to $1000 \mathrm{mg} /$ day administered for 3-5 consecutive days, the dose was established according to the presence or not of other systemic manifestations apart from uveitis. All of them had active intraocular inflammation at the moment of the study. BCVA values improved considerably after 1 month (Figure 1). No major side effects were observed.

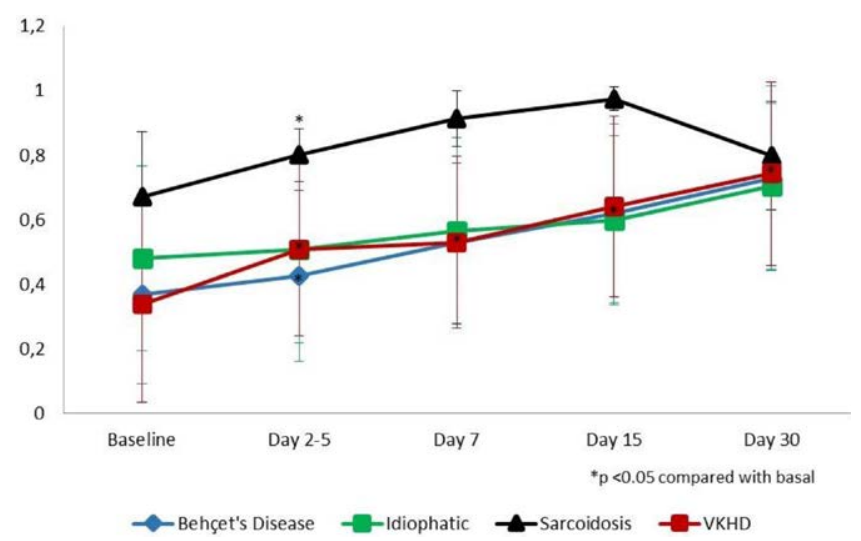

Figure 1. Improvement of best corrected visual acuity (BCVA).
Conclusion: High-dose IVMP pulse therapy is useful and safe for a prompt control of BCVA regardless of the underlying IMID.

\section{REFERENCES:}

[1] Vegas-Revenga N, et al. Am J Ophthalmol. 2019; 200:85-94. doi: 10.1016/j. ajo.2018.12.019

[2] Calvo-Río V, et al. Clin Exp Rheumatol. 2014;32(4 Suppl 84): S54-7. PMID: 25005576

[3] Santos-Gómez M, et al. Clin Exp Rheumatol. 2016;34(6 Suppl 102): S34S40. PMID:27054359

[4] Atienza-Mateo B, et al. Rheumatology (Oxford) 2018;57(5):856-864. doi: 10.1093/rheumatology/kex480.

[5] Atienza-Mateo B, et al. Arthritis Rheumatol. 2019; 71(12):2081-2089. doi 10.1002/art.41026.

[6] Martín-Varillas JL, et al. Ophthalmology. 2018;125(9):1444-1451. doi 10.1016/j.ophtha.2018.02.020

Table 1. Main features of 71 patients with NIU. Data are of affected eyes.

\begin{tabular}{|c|c|c|c|c|c|}
\hline & VKHD & Idiophatic & Behcet's & Sarcoidosis & s Overall \\
\hline & $(n=24)$ & $(n=23)$ & $(n=19)$ & $(n=5)$ & $(n=71)$ \\
\hline Men/Women, $\mathrm{n}$ & $5 / 19$ & $9 / 14$ & $9 / 10$ & $2 / 3$ & 71 \\
\hline Mean age (years) $\pm \mathrm{SD}$ & $42 \pm 11$ & $47 \pm 15$ & $33 \pm 10$ & $42 \pm 22$ & - \\
\hline $\begin{array}{l}\text { Unilateral/Bilateral } \\
\text { NIU, n (\%) }\end{array}$ & $2(8.3) / 22(91.7)$ & $10(43.5) / 13(56.5)$ & $4(21) / 15(79)$ & $3(60) / 2(40)$ & $19 / 52$ \\
\hline \multicolumn{6}{|l|}{ NIU patterns, n (\%) } \\
\hline Posterior uveitis & $6(25)$ & $9(39.1)$ & $3(15.8)$ & $1(20)$ & 19 \\
\hline Panuveitis & $18(75)$ & $14(60.9)$ & $16(84.2)$ & $4(80)$ & 52 \\
\hline \multicolumn{6}{|c|}{ Laboratory data, $\mathrm{n}(\%)$} \\
\hline ANA & $2(8.34)$ & $2(8.7)$ & $0(0)$ & $1(20)$ & 5 \\
\hline HLA B27 & $0(0)$ & $4(17.4)$ & $0(0)$ & $0(0)$ & 4 \\
\hline HLA B29 & $0(0)$ & $1(4.3)$ & $0(0)$ & $0(0)$ & 1 \\
\hline HLA B51 & $0(0)$ & $5(21.7)$ & $8(42)$ & $3(60)$ & 16 \\
\hline Angiotensin & $1(4.17)$ & $2(8.7)$ & $0(0)$ & $1(20)$ & 4 \\
\hline $\begin{array}{l}\text { Converting Enzyme } \\
\text { (ACE) }\end{array}$ & & & & & \\
\hline
\end{tabular}

Disclosure of Interests: None declared

DOI: 10.1136/annrheumdis-2021-eular.2159

\section{AB0772 $\quad$ JAK INHIBITORS IN REFRACTORY ADULT AND CHILDHOOD ONSET STILL'S DISEASE}

L. Gillard ${ }^{1}$, S. Mitrovic ${ }^{1,2}$, H. Reumaux ${ }^{3}$, M. Michaud ${ }^{4}$, F. Cohen ${ }^{1,2}$, J. Pouchot $^{5}$

B. Fautrel ${ }^{1,2,6}$. ${ }^{1}$ Pitié-Salpêtrière, AP-HP, Rheumatology Department, Paris,

France; ${ }^{2}$ Sorbonne Université, Rheumatology Department, Paris, France;

${ }^{3}$ Jeanne de Flandre Hospital, Rheumatology, Lille, France; ${ }^{4}$ Hospital Joseph

Ducuing, Medecine Interne, Toulouse, France; ${ }^{5}$ European Hospital Georges

Pompidou, Medecine Interne, Paris, France: ${ }^{6}$ Pierre Louis Institute of

Epidemiology and Public Health, INSERM UMR 1136, Paris, France

Background: Excessive and inappropriate production of pro-inflammatory cytokines such as interleukin (IL)-1, IL-6 or IL-18, is a pathogenic cornerstone in adult and childhood onset Still's disease. Beyond therapies targeting IL-1 or IL-6, Janus kinases (JAK) inhibitors have been proposed for adult-onset Still's disease (AOSD) patients refractory to or intolerant of treatment with biologicals. Recently, it has been suggested that JAK inhibitors might be efficient in refractory AOSD patients ${ }^{1}$.

Objectives: To assess the efficacy and safety of JAK inhibitors in the treatment of refractory systemic juvenile idiopathic arthritis (SJIA) or AOSD.

Methods: This retrospective study was based on a national survey of the departments of rheumatology, paediatric rheumatology and internal medicine in al French hospitals from an online call of the "Club Rhumatismes et Inflammation" (www.cri-net.com). The data were collected using a standardized questionnaire, and analyzed at different time points (treatment initiation, M1, M3, M6 and end of the follow-up). The response to JAK inhibitors was categorized as: complete remission (resolution of all clinical and biologic signs), partial remission (clinical improvement with persistence of a few symptoms) or failure (lack of clinical or biological improvement).

Results: 6 patients ( 5 adults and 1 child) were recruited (Table 1 ). Mean age at treatment start was 39.6 years for the AOSD patient and 6 years for the SJIA patient, and mean disease duration was 5.3 years. The clinical expression was predominantly systemic in 5 five patients and chronic articular in one. Response to corticosteroids, conventional synthetic or biological Disease Modifying Anti-Rheumatic Drugs had been considered inadequate in all patients. Baricitinib was used in 3 patients, ruxolitinib in 2 , and tofacitinib in 1. Steroids were concurrently used in all patients, anakinra in one, methotrexate and anakinra in one. At a mean (SD) follow-up of 9.5 months, partia response was observed in $4(66.7 \%)$ cases (patients with ruxolitinib, tofacitinib 